
\section{(3)}

\title{
Comparison of antimicrobial activity between intraoperatively soaked bacitracin sutures and triclosan coated suture
}

Shane T. Musick ${ }^{1 *}$, Jeremy M. Adkins ${ }^{1 \uparrow}$, Roy Al-Ahmar ${ }^{2 \&}$, Hongwei D. Yu²,3\&, Anthony M. Alberico $^{1 \&}$

${ }^{1}$ Department of Neurosurgery, Marshall University Joan C. Edwards School of Medicine, Huntington, WV, United States of America

${ }^{2}$ Department of Biomedical Sciences, Marshall University Joan C. Edwards School of Medicine, Huntington, WV, United States of America

${ }^{3}$ Department of Pediatrics, Marshall University Joan C. Edwards School of Medicine, Huntington, WV, United States of America

*Corresponding Author:

Shane.musick@gmail.com (SM)

TThese authors contributed equally to this work.

\&These authors also contributed equally to this work.

\section{Short Title: Intraoperatively Prepared Bacitracin Suture}

\section{Keywords}

Surgical site infection, antimicrobial suture, intraoperative

0

1




\section{Abstract}

With the easily available option for surgeons to soak their suture in anti-biotic irrigating

45 solution intraoperatively in mind, this study was designed to evaluate the ability of suture soaked

46 in bacitracin irrigating solution to inhibit the growth of Staphylococcus aureus and Methicillin-

47 resistant Staphylococcus aureus. Using standard experimental procedure, sterile suture was

48 soaked in Bacitracin suture, and dried for 10 minutes or 6 hours, incubated for $24 \mathrm{~h}$ on inoculated

49 plates, and examined for zone of inhibition around the suture. This was compared to control

50 unsoaked suture and antimicrobial suture (AMS) currently on the market to determine if the

51 minor intra operative procedural change of placing suture in antibiotic irrigation solution instead

52 of on the sterile table could confer some antimicrobial activity. The study found the Bacitracin

53 soaked suture (BSS) consistently inhibited the growth of the test organisms. For both organisms,

54 the BSS exhibited a significantly larger zone of inhibition compared to the unsoaked control

55 suture. However, the AMS currently on the market exhibited a larger zone of inhibition

56 compared to the BSS. Placing sutures in a bacitracin irrigation solution intraoperatively instead

57 of directly on the sterile table can achieve some of the in vitro antimicrobial effect seen from

58 AMS currently on the market. This may result in reduced rates of SSIs and associated costs

59 without major procedural change and at reduced overhead.

\section{Introduction}

$$
\text { Surgical site infections (SSIs) can be a complication of any surgical procedure and, as }
$$

62 such, antimicrobial suture (AMS) represents a strategy developed in an effort to mitigate this

63 risk. Some studies have suggested that AMS reduces SSIs in a wide range of procedures (1-9).

64 Yet, others have suggested they have no such role $(10,11)$. Currently, the role of AMS in 
65 reducing SSIs remains controversial. Furthermore, early data has largely focused on their

66 application for gastrointestinal (GI) procedures, but with recent studies supporting their utility,

67 others are exploring their potential utility in ophthalmology and orthopedics $(12,13)$. Thus, the

68 increasing evidence that AMS play a role in preventing SSIs could eventually lead to higher

69 utilization rates in procedures across specialties.

The aim of this study is to determine whether or not the practice of simply having the OR

72 technician place the closing suture in the bacitracin irrigation solution at the onset of the case

73 rather than setting it on the OR table could result in the conveyance of increased antimicrobial

74 activity to the suture material.

75 Methods

\section{Suture Preparation}

A vial of Bacitracin powder (Pfizer Inc., New York) was reconstituted using sterile

78 technique with $0.9 \% \mathrm{NaCl}$ Irrigation, USP (Aqualite, Illinois) to create a Bacitracin solution with

79 a concentration of 1000 Units/mL to replicate the intraoperative bacitracin irrigation solution

80 concentration typically used intraoperatively. Ethicon (Somerville, NJ) 0 Vicryl was then placed

81 in this Bacitracin solution and soaked for 1 hour to replicate intraoperative soaking time. This is

82 shown in figure 1. This Bacitracin soaked suture (BSS) was then placed in a sterile petri dish at

83 room temperature to dry — one group for 10 minutes to replicate intraoperative drying time

84 outside of the solution and a second group for 6 hours to ensure that any measured antimicrobial

85 activity was provided by the prepared suture, not excess Bacitracin solution.

86 Fig 1. Ethicon 0 VICRYL* sutures soaking in bacitracin irrigation solution

87 intraoperatively. This displays the ease and feasibility of placing suture packs into an

88 antimicrobial solution during the course of the procedure. 


\section{Measuring Antibacterial Activity}

Using standard experimental procedure, Staphylococcus aureus (ATCC 6538) and

92 Methicillin Resistant Staphylococcus aureus (MRSA) (N315) were grown in Luria Broth (LB)

93 (Difco) broth at $37^{\circ} \mathrm{C}$ to a MacFarland Turbidity of $1\left(5 \times 10^{8} \mathrm{CFU} / \mathrm{ml}\right)$. Subsequently, 100ul of

94 each broth was then inoculated on 27 separate 1.5\% agar plates. For each group of 27 agar

95 plates: 3 were plated with one $\mathrm{cm}$ of the unsoaked Ethicon 0 VICRYL ${ }^{\circledR}$ Suture as a control, 6

96 with a $10 \mu \mathrm{l}$ aliquot of the $1000 \mathrm{U} / \mathrm{mL}$ Bacitracin solution as an additional control, 6 with one $\mathrm{cm}$

97 sections of BSS dried for 10 minutes, 6 with one $\mathrm{cm}$ sections of BSS dried for 6 hours, and 6

98 with one cm sections of the Ethicon Coated 0 VICRYL ${ }^{\circledR}$ Plus Antibacterial (polyglactin 910).

99 The growth plates were subsequently incubated at $37^{\circ} \mathrm{C}$ for 24 hours, after which they were

100 examined for the presence or absence of a zone of inhibition.

\section{Statistical Analysis}

Statistical analysis was performed using GraphPad Prism 7.02 (GraphPad Software,

103 California). A one-way ANOVA was used followed by Tukey's multiple comparison test to

104 evaluate the mean difference of the inhibition zone. Student's t-test was used for individual

105 comparisons. Statistical significance was determined at $\mathrm{P}<0.05$.

\section{Results}

The size results of the zone of inhibition assays are listed in Table 1. A zone of inhibition

108 was present for all replicates of the Bacitracin solution aliquot, both drying times of the BSS, and

109 Ethicon Coated 0 VICRYL ${ }^{\circledR}$ Plus Antibacterial suture. As expected, the non-antibiotic Ethicon 0

110 Vicryl failed to exhibit any zone of inhibition. Typical zones of inhibition are shown in Figure 2. 
111 Table 1. Comparison of zone of inhibition of Staphylococcus aureus and Methicillin

112 Resistant Staphylococcus aureus (MRSA).

\begin{tabular}{|c|c|c|c|c|c|}
\hline & $\begin{array}{c}0 \text { VICRYL } \\
\end{array}$ & Bacitracin & 10 Minute & 6 Hour & Coated 0 VICRYL ${ }^{\circledR}$ \\
\hline Zone of & & Solution Aliquot & Dried BSS ${ }^{\circledR}$ & Dried BSS ${ }^{\mathrm{a}}$ & Plus Antibacterial \\
Inhibition v. & $0 \mathrm{~mm}$ & $18.2 \mathrm{~mm} \pm 0.84$ & $7 \mathrm{~mm} \pm 1.41$ & $7 \mathrm{~mm} \pm 1.79$ & $19.5 \mathrm{~mm} \pm 2.07$ \\
S. aureus & & & & & \\
\hline Zone of & & & & & \\
Inhibition v. & $0 \mathrm{~mm}$ & $15.5 \mathrm{~mm} \pm 1.05$ & $5 \mathrm{~mm} \pm 1.22$ & $6.83 \mathrm{~mm} \pm 0.75$ & $17.67 \mathrm{~mm} \pm 1.37$ \\
MRSA & & & & & \\
\hline
\end{tabular}

This displays the differing sizes of zones of microbial inhibition across the suture trials. From left to right comparisons - sutures devoid of any bacitracin solution, an aliquot of bacitracin solution itself, 10-minute dried BSS sutures, 6-hour dried BSS sutures, and the pre-coated antibacterial suture.

${ }^{\text {aBSS }}=$ Bacitracin solution-soaked Ethicon 0 VICRYL ${ }^{\circledR}$ Suture

Figure 2: Zone of inhibition petri dish images

A: Image showing (from left to right) Staphylococcus aureus in LB broth grown on $1.5 \%$ agar plates grown in the presence of $10 \mu \mathrm{l}$ aliquot of $1000 \mathrm{U} / \mathrm{ml}$ Bacitracin solution, 0 Vicryl ${ }^{\circledR}$ Suture,

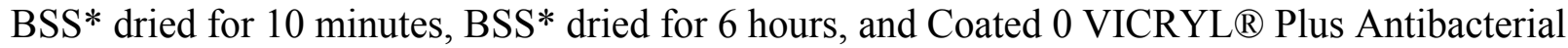
after incubation at $37 \mathrm{oC}$ for 24 hours. B: Image showing (from left to right) MRSA in LB broth grown on $1.5 \%$ agar plates grown in the presence of $10 \mu \mathrm{l}$ aliquot of $1000 \mathrm{U} / \mathrm{ml}$ Bacitracin solution, 0 Vicryl® Suture, BSS* dried for 10 minutes, BSS* dried for 6 hours, and Coated 0 VICRYL $\AA$ Plus Antibacterial after incubation at $37^{\circ} \mathrm{C}$ for 24 hours. different drying times using BSS. There were no significant differences detected between

131 Ethicon Coated 0 VICRYL® Plus Antibacterial suture and the Bacitracin solution aliquot

$132(\mathrm{p}=0.58)$. For $\mathrm{S}$. aureus and MRSA, both drying times of the BSS exhibited a significantly larger 133 zone of inhibition than the control unsoaked Ethicon 0 Vicryl $(\mathrm{p}<0.0001)$. However, for $\mathrm{S}$. 
aureus and MRSA, the Ethicon Coated 0 VICRYL ${ }^{\circledR}$ Plus Antibacterial suture and Bacitracin

135 solution aliquot had significantly larger zone of inhibitions than both drying times of the BSS (p

$136<0.0001)$. Overall, these results demonstrate that both drying times are similarly effective,

137 suggesting feasibility of use throughout the entirety of a procedure. The BSS displays robust and

138 statistically significant zones of inhibition, indicating antibacterial activity. The zones of

139 inhibition of the Ethicon Coated 0 VICRYL ${ }^{\circledR}$ Plus Antibacterial suture was statistically

140 significantly larger than the BSS groups.

\section{Discussion}

143 triclosan sutures in 2002, their reception has been mixed with recent trials and meta-analyses

144 providing evidence of their benefit (1-9), despite individual studies initially failing to show a

145 reduction in SSIs $(10,11)$. In vitro studies of AMS have provided further support for their

146 benefit. Rothenburger et al. used zone of inhibition assays to demonstrate that polyglactin 910

147 with triclosan sutures inhibit the growth of S. aureus and S. epidermidis even after passes

148 through tissue and aqueous immersion (14). Edmiston et al. demonstrated decreased adherence of

149 Gram positive and negative bacterial species to AMS which lead to decreased local bacterial

150 loads for at least up to 96 hours (15). This latter finding could help explain the findings of a

151 study by Ford et al. that reported less postoperative pain in general pediatric surgical patients in

152 which AMS were used, although interestingly it failed to show a concomitant decrease in SSIs

153 within the group (16).

154 Most initial studies have investigated the utility of AMS in GI procedures, but recent

155 studies have begun to explore broader applications. Rozzelle et al. showed decreased CSF shunt

156 infection rates with the use of AMS (8). Others have demonstrated decreased bacterial loads 
157 around implant sites in rat models, suggesting a role for AMS in decreasing implant infections

158 (13). With these added applications, others have attempted to develop sutures for

159 ophthalmological procedures (12).

160 Despite the increased upfront costs, AMS have demonstrated their cost effectiveness by

161 decreasing overall costs related to the management of wound infections. One study estimated the

162 widespread use of AMS at one center could save approximately $\$ 1.5$ million in a year by

163 avoiding costs related to SSIs (3). Additionally, another study found AMS to significantly reduce

164 the incidence of SSIs and subsequently lead to reduced overall costs as its analysis found the

165 average cost of SSI management to be $\$ 2,310(7)$.

Given the reduction in SSIs and subsequent associated costs, our study sought to replicate

167 the antimicrobial activity of marketed AMS at a reduced overhead by having the surgical

168 technician place the sutures in a bacitracin solution that is prepared at the beginning of the

169 procedure for use as irrigation in lieu of directly placing them on the table. An example of this

170 procedural change is shown in Figure 1. Our in vitro antimicrobial zone of inhibition assays

171 suggests that our intraoperatively prepared Ethicon 0 VICRYL $®$ BSS provide superior

172 antimicrobial activity compared to unsoaked Ethicon 0 VICRYL ${ }^{\circledR}$ suture. This benefit is

173 potentially due to a Bacitracin coating that forms during the soaking process, evinced by

174 sustained antimicrobial activity even after allowing 6 hours of drying. Yet, our study also

175 suggests that while the soaking process confers some antimicrobial activity, it fails to retain the

176 full antimicrobial exhibited by an aliquot of the Bacitracin irrigation solution itself. Additionally,

177 the current AMS on the market-Ethicon's Coated 0 VICRYL ${ }^{\circledR}$ Plus Antibacterial suture-

178 exhibits superior antimicrobial activity to our intraoperatively BSS, likely due to enhanced drug-

179 eluting properties related to their manufacturing process. However, as zones of inhibition signify 
180 drug potency, the differing sizes may not be clinically relevant. The BSS displays a consistent

181 and robust antibacterial activity, with the Ethicon Coated 0 VICRYL ${ }^{\circledR}$ Plus Antibacterial suture

182 demonstrating a greater drug concentration and eluting property as evinced by the greater zone of

183 inhibition sizes. Therefore, given local potency of the BSS in preventing bacterial growth, there

184 is doubtful to be clinically meaningful differences in SSI incidence using either suture. Further

185 studies examining the antimicrobial activity of our BSS after passes through tissue and aqueous

186 immersion could help identify the longevity and overall viability of this antimicrobial coating in

187 a clinical setting.

188 Ultimately, this study suggests that with the minor procedural change of placing sutures

189 in a bacitracin irrigation solution intraoperatively instead of directly on the table, it can achieve

190 some of the antimicrobial effect of the AMS currently on the market. This may result in reduced

191 rates of SSIs and associated costs without major procedural change and at reduced overhead.

192 Future studies should be undertaken utilizing this change to fully ascertain its clinical efficacy

193 and price-reducing effectiveness.

\section{Conclusions}

AMS lower the incidence of SSI and subsequently decrease associated costs despite

196 increased overhead cost. We attempted to develop a novel method that could reproduce the

197 antimicrobial benefits while lowering these overhead costs. Our zone of inhibition assays

198 suggests that our method of intraoperatively soaking sutures in the antibiotic irrigation confers

199 antimicrobial activity against S. aureus and MRSA that is superior to plain suture, yet inferior to

200 the AMS currently on the market. Further studies are needed to determine if our BSS can better

201 replicate the antimicrobial activity of the AMS currently on the market and if the antimicrobial

202 activity observed in our study has clinical implications. 


\section{References}

204

205

206

207

208

209

210

211

212

213

214

215

216

217

218

219

220

221

222

223

224

225

226

227

228

229

230

231

232

233

234

235

236

237

238

239

240

241

242

243

244

245
1. Daoud FC, Edmiston CE, Jr., Leaper D. Meta-analysis of prevention of surgical site infections following incision closure with triclosan-coated sutures: robustness to new evidence. Surg Infect (Larchmt). 2014;15(3):165-81.

2. Edmiston CE, Jr., Daoud FC, Leaper D. Is there an evidence-based argument for embracing an antimicrobial (triclosan)-coated suture technology to reduce the risk for surgicalsite infections?: A meta-analysis. Surgery. 2013;154(1):89-100.

3. Galal I, El-Hindawy K. Impact of using triclosan-antibacterial sutures on incidence of surgical site infection. Am J Surg. 2011;202(2):133-8.

4. Guo J, Pan LH, Li YX, Yang XD, Li LQ, Zhang CY, et al. Efficacy of triclosan-coated sutures for reducing risk of surgical site infection in adults: a meta-analysis of randomized clinical trials. J Surg Res. 2016;201(1):105-17.

5. Justinger C, Slotta JE, Ningel S, Graber S, Kollmar O, Schilling MK. Surgical-site infection after abdominal wall closure with triclosan-impregnated polydioxanone sutures: results of a randomized clinical pathway facilitated trial (NCT00998907). Surgery. 2013;154(3):589-95. 6. Leaper D, Wilson P, Assadian O, Edmiston C, Kiernan M, Miller A, et al. The role of antimicrobial sutures in preventing surgical site infection. Ann R Coll Surg Engl. 2017;99(6):439-43.

7. Nakamura T, Kashimura N, Noji T, Suzuki O, Ambo Y, Nakamura F, et al. Triclosancoated sutures reduce the incidence of wound infections and the costs after colorectal surgery: a randomized controlled trial. Surgery. 2013;153(4):576-83.

8. Rozzelle CJ, Leonardo J, Li V. Antimicrobial suture wound closure for cerebrospinal fluid shunt surgery: a prospective, double-blinded, randomized controlled trial. J Neurosurg Pediatr. 2008;2(2):111-7.

9. Wang ZX, Jiang CP, Cao Y, Ding YT. Systematic review and meta-analysis of triclosancoated sutures for the prevention of surgical-site infection. Br J Surg. 2013;100(4):465-73.

10. Diener MK, Knebel P, Kieser M, Schuler P, Schiergens TS, Atanassov V, et al.

Effectiveness of triclosan-coated PDS Plus versus uncoated PDS II sutures for prevention of surgical site infection after abdominal wall closure: the randomised controlled PROUD trial. Lancet. 2014;384(9938):142-52.

11. Elsolh B, Zhang L, Patel SV. The Effect of Antibiotic-Coated Sutures on the Incidence of Surgical Site Infections in Abdominal Closures: a Meta-Analysis. J Gastrointest Surg. 2017;21(5):896-903.

12. Kashiwabuchi F, Parikh KS, Omiadze R, Zhang S, Luo L, Patel HV, et al. Development of Absorbable, Antibiotic-Eluting Sutures for Ophthalmic Surgery. Transl Vis Sci Technol. 2017;6(1):1.

13. Marco F, Vallez R, Gonzalez P, Ortega L, de la Lama J, Lopez-Duran L. Study of the efficacy of coated Vicryl plus antibacterial suture in an animal model of orthopedic surgery. Surg Infect (Larchmt). 2007;8(3):359-65.

14. Rothenburger S, Spangler D, Bhende S, Burkley D. In vitro antimicrobial evaluation of Coated VICRYL* Plus Antibacterial Suture (coated polyglactin 910 with triclosan) using zone of inhibition assays. Surg Infect (Larchmt). 2002;3 Suppl 1:S79-87. 
246 15. Edmiston CE, Seabrook GR, Goheen MP, Krepel CJ, Johnson CP, Lewis BD, et al. 247 Bacterial adherence to surgical sutures: can antibacterial-coated sutures reduce the risk of 248 microbial contamination? J Am Coll Surg. 2006;203(4):481-9.

249 16. Ford HR, Jones P, Gaines B, Reblock K, Simpkins DL. Intraoperative handling and 250 wound healing: controlled clinical trial comparing coated VICRYL plus antibacterial suture 251 (coated polyglactin 910 suture with triclosan) with coated VICRYL suture (coated polyglactin 252910 suture). Surg Infect (Larchmt). 2005;6(3):313-21. 


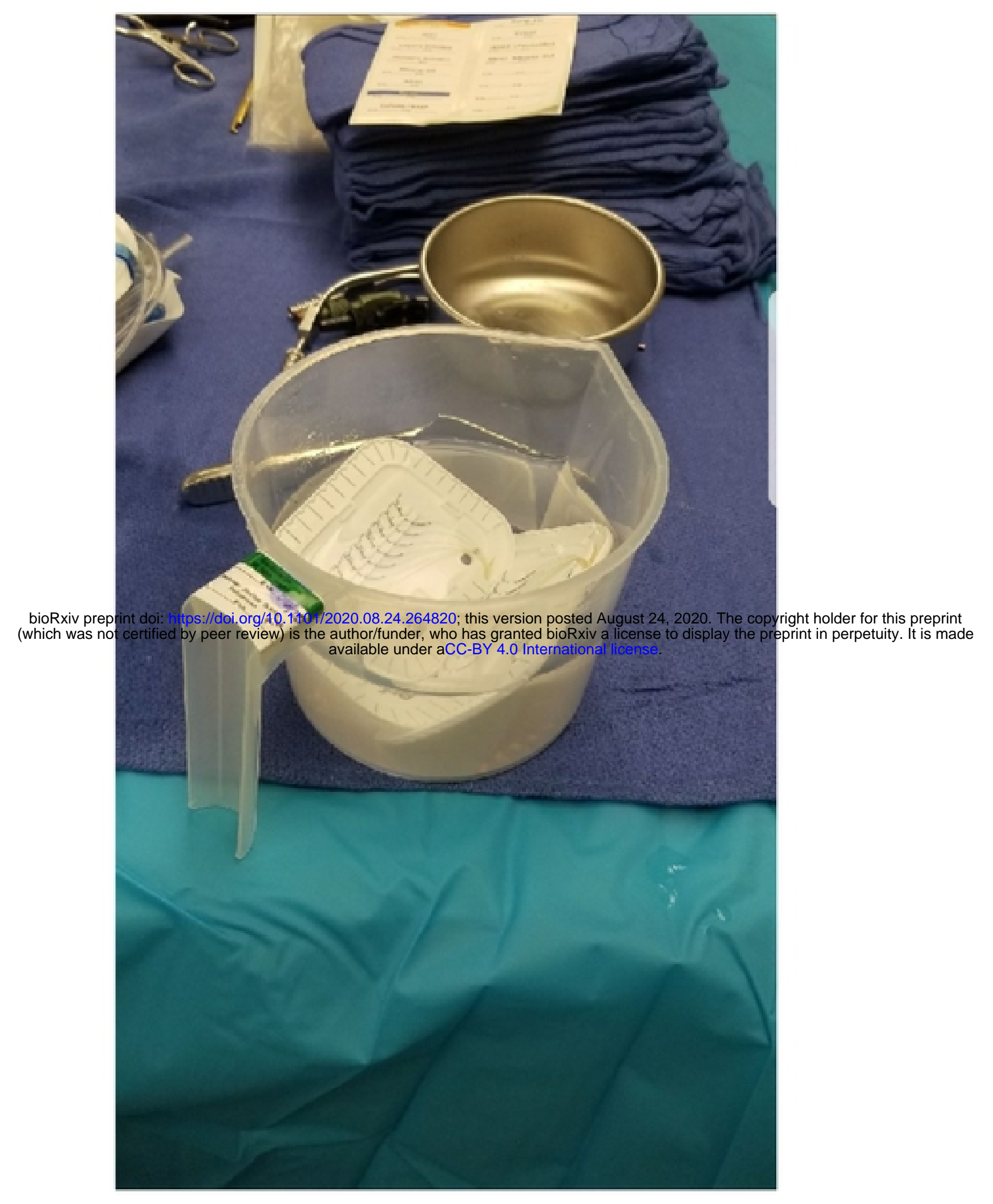

Figure 1 


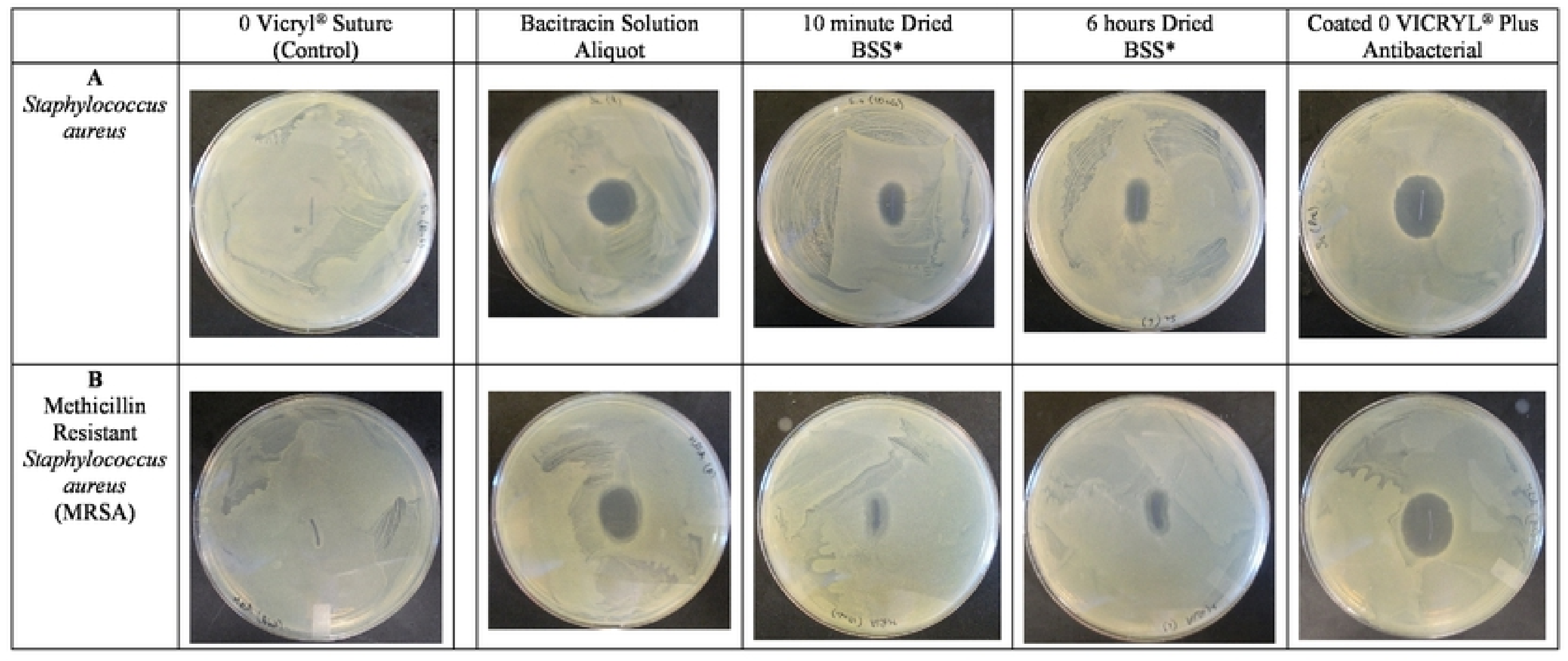

Figure 2 